\title{
Spacecraft Constrained Maneuver Planning for Moving Debris Avoidance Using Positively Invariant Constraint Admissible Sets
}

\author{
Avishai Weiss, Morgan Baldwin, R. Scott Erwin and Ilya Kolmanovsky
}

\begin{abstract}
This paper extends our prior work on spacecraft debris avoidance based on positively invariant sets to the case of moving debris. In this approach, a timevarying connectivity graph is constructed between a set of forced equilibria, forming a virtual net that is centered around a nominal orbital position of the spacecraft. The connectivity between two equilibria is determined based on contractive sets in order to guarantee that collisionfree transitions between equilibria can be effected within a giving time period. A graph search algorithm is implemented to find the shortest path around the debris, or through the debris' path once it has moved on. Simulation results are presented that illustrate this approach.
\end{abstract}

\section{INTRODUCTION}

Given the rapidly growing amount of debris in Earth orbit, spacecraft maneuvers must satisfy debris avoidance requirements. While obstacle avoidance has been studied extensively in robotics applications [3], [4], the related spacecraft problems have several unique features and benefit from the development of specialized approaches and algorithms. In particular, the space environment is relatively uncluttered. Spacecraft dynamics are different from those of typical robots. Maneuver efficiency with respect to time and fuel consumption is important. The states of the spacecraft and the debris can only be estimated, often with a significant estimation error. Finally, computational algorithms must be suitable for implementation on-board where computing power is very limited.

In [1], we have introduced an on-board maneuver planning approach based on the use of constraintadmissible positively invariant sets to determine connectivity between a set of forced and unforced spacecraft equilibria forming a virtual net in the vicinity of

A. Weiss is a Graduate Student, Department of Aerospace Engineering, The University of Michigan, Ann Arbor, MI.

M. Baldwin is a Research Aerospace Engineer, Space Vehicles Directorate, Air Force Research Laboratory, Kirtland Air Force Base, NM.

R. Scott Erwin is a Principal Research Aerospace Engineer, Space Vehicles Directorate, Air Force Research Laboratory, Kirtland Air Force Base, NM.

I. Kolmanovsky is a Professor, Department of Aerospace Engineering, The University of Michigan, Ann Arbor, MI. the spacecraft. Two equilibria are connected if a choice of a Linear Quadratic (LQ) feedback gain can be made that results in a transition between the equilibria and which avoids the debris collision while satisfying the imposed thrust limits. The connectivity graph for all the equilibria in the net is constructed and graph search algorithms are employed to optimize an equilibria hopping sequence that avoids the debris collisions.

Unlike the open-loop trajectory optimization approaches, we do not rely on precise assignment of spacecraft position to the time instants along the trajectory, but instead switch to the next set-point and controller gain once appropriate conditions are satisfied. While this approach can be conservative, it facilitates fault-tolerant and disturbance-tolerant execution of the maneuvers. Furthermore, by employing disturbanceinvariant sets [14], we can assure robustness to unmeasured (but set-bounded) disturbances and uncertainties. This extension to handling unmeasured disturbances and uncertainties using techniques of [14] is not pursued in the present paper.

To facilitate the on-board construction of the connectivity graph, a fast growth distance computation procedure between two ellipsoidal sets has been proposed in [1]. In this approach, using the Karush-Kuhn-Tucker conditions, the growth distance computations are reduced to a root finding problem for the scalar value of the Lagrange multiplier. Then a predictor-corrector dynamic Newton-Raphson algorithm is used to update the Lagrange multiplier thereby rapidly estimating the growth distance from different equilibria in the virtual net to the debris.

In [2], we incorporated limited thrust requirements into the computation of thrust limit on the growth distance. Even though the computation of thrust limits on the growth distance can be performed offline for the nominal operating conditions, fast computational procedures are beneficial in case of thruster failures, degradations, and restrictions on thrust directions (e.g., caused by the presence of other spacecraft nearby), all of which can lead to changing constraints on thrust during spacecraft missions. If the thrust limits are prescribed in the form of 2-norm bounds, the optimization 
problem involved in computing the thrust limit on the growth distance is non-convex (unlike computing the growth distance itself). Consequently, we used polyhedral norm bounds on thrust, that lent themselves to explicit solutions.

In this paper, we address the problem of avoiding a moving debris. Our connectivity graph construction utilizes the sets, centered about the equilibria in the virtual net, that are guaranteed to contract to specified smaller subsets in $N$ steps. The connectivity of two equilibria in the net ensures that over $N$ steps the transition to the neighborhood of the target equilibrium (i.e., smaller subset) can occur without the debris colliding with the spacecraft. The graph vertices are formed based on equilibria and the control time intervals of $N$ steps. A graph search algorithm is employed to determine a feasible debris avoidance trajectory.

The related literature on spacecraft trajectory optimization with obstacle/debris avoidance is surveyed in [1]. Previous research addresses topics in spacecraft trajectory optimization [6], collision avoidance strategies based on risk assessment [7], the use of artificial potential functions [8], [9], and the use of conventional and mixed integer linear programming techniques [10], [11], [12], [13].

The paper is organized as follows. In Section II we discuss the nonlinear and the linearized models used to represent spacecraft relative motion dynamics. In Section III we review our approach to constructing the virtual net based on a set of forced and unforced equilibria, and using this virtual net for debris avoidance. The procedure to compute the thrust limit on the growth distance is presented. Simulation results are reported in Section IV. Finally, concluding remarks are made in Section V.

\section{Relative Motion Model}

The spacecraft relative motion model represents the spacecraft dynamics in the (non-inertial) Hill's frame with the origin at a target location on a nominal circular orbit. For small distances, the linearization of the relative motion model given by the Hill-ClohessyWiltshire $(\mathrm{CWH})$ equations is used [15].

\section{A. Nonlinear equations of motion}

The relative position vector of the spacecraft with respect to a target location on a circular orbit is expressed as

$$
\delta \vec{r}=x \hat{\imath}+y \hat{\imath}+z \hat{k},
$$

where $x, y$ and $z$ are the components of the position vector of the spacecraft relative to the target location and $\hat{\imath}, \hat{\jmath}, \hat{k}$ are the unit vectors of the Hill's frame. The Hill's frame has its $x$-axis along the orbital radius, $y$ axis along the orbital track, and $z$-axis is orthogonal to orbital plane.

The position vector of the spacecraft with respect to the center of the Earth can be expressed as $\vec{R}=$ $\vec{R}_{0}+\delta \vec{r}=\left(R_{0}+x\right) \hat{\imath}+y \hat{\jmath}+z \hat{k}$, where $R_{0}$ is the nominal orbital radius. The nonlinear equations of motion for the spacecraft (relative to an inertial frame) can be expressed in vector form as

$$
\ddot{\vec{R}}=-\mu \frac{\vec{R}}{R^{3}}+\frac{1}{m_{c}} \vec{F},
$$

where $\vec{F}$ is the vector of external forces applied to the spacecraft, $R=|\vec{R}|, m_{c}$ is the mass of the spacecraft, $\mu$ is the gravitational constant, and

$$
\ddot{\vec{R}}=\left(\ddot{x}-2 n \dot{y}-n^{2} x\right) \hat{\imath}+\left(\ddot{y}+2 n \dot{x}-n^{2} y\right) \hat{\jmath}+(\ddot{z}) \hat{k} .
$$

In these equations, $n=\sqrt{\frac{\mu}{R_{0}^{3}}}$ denotes the mean motion on the nominal orbit. Similar equations can be used to describe the motion of the debris.

\section{B. Linearized HCW equations in discrete-time}

For $\delta r<<R$, the linearized Hill-Clohessy-Wiltshire (HCW) equations [15] approximate the relative motion of the spacecraft on a circular orbit as

$$
\begin{gathered}
\ddot{x}-3 n^{2} x-2 n \dot{y}=\frac{F_{x}}{m_{c}}, \\
\ddot{y}+2 n \dot{x}=\frac{F_{y}}{m_{c}}, \\
\ddot{z}+n^{2} z=\frac{F_{z}}{m_{c}},
\end{gathered}
$$

where $F_{x}, F_{y}, F_{z}$ are components of the external force vector (excluding gravity) acting on the spacecraft. The linearized dynamics account for differences in gravity between the spacecraft and nominal orbital location, and for relative motion effects. The spacecraft relative motion dynamics in the orbital plane ( $x$ and $y$ ) and in the out-of-orbital plane $(z)$ are decoupled. The inplane dynamics are Lyapunov unstable, while the outof-plane dynamics are Lyapunov stable. The in-plane dynamics are completely controllable from $F_{y}$ input but are not controllable from $F_{x}$ input. The out-ofplane dynamics are controllable from $F_{z}$ input. These dynamics are clearly different from typical ground robots.

Assuming a sampling period of $\Delta T$ sec, we can convert the model (2) to a discrete-time form

$$
X(t+1)=A X(t)+B U(t),
$$


where $X(t)=[x(t), y(t), \quad z(t), \dot{x}(t), \quad \dot{y}(t), \dot{z}(t)]^{\mathrm{T}}$ is the state vector at the time instant $t \in Z^{+}$, $U(t)=\left[F_{x}(t), F_{y}(t), F_{z}(t)\right]^{\mathrm{T}}$ is the control vector of thrust forces at the time instant $t \in Z^{+}$, and $A=$ $\exp \left(A_{c} \Delta T\right), B=\int_{0}^{\Delta T} \exp \left(A_{c}(\Delta T-\tau)\right) d \tau B_{c}$ are the discretized matrices obtained based on the continuoustime system realization $\left(A_{c}, B_{c}\right)$ in (2). Alternatively, the control vector $U$ can represent an instantaneous change in the velocity of the spacecraft, $\Delta v$, induced by thrust, with an appropriately re-defined $B$-matrix,

$$
B_{\Delta v}=e^{A_{c} \Delta T}\left[\begin{array}{ccc}
0 & 0 & 0 \\
0 & 0 & 0 \\
0 & 0 & 0 \\
1 & 0 & 0 \\
0 & 1 & 0 \\
0 & 0 & 1
\end{array}\right]
$$

\section{Debris AVoidance Based on A Virtual NET}

Our approach to debris avoidance is based on utilizing constraint-admissible positively invariant sets [14], [16] centered around the spacecraft forced and unforced relative motion equilibria. A finite set of these equilibria used for constructing debris avoidance maneuvers is referred to as a virtual net. Given an estimate of the debris position, we build a connectivity graph that identifies the equilibria in the virtual net between which the spacecraft can move, with guaranteed collision-free motion and within the available thrust authority. We then employ graph search to determine an efficient path between the equilibria that ensures debris avoidance.

\section{A. Virtual Net}

The virtual net comprises a finite set of equilibria, $X_{e}(r)$, corresponding to a finite set of prescribed spacecraft positions $r \in \mathcal{N}=\left\{r_{1}, r_{2}, \ldots, r_{n}\right\} \subset R^{3}$,

$$
X_{e}\left(r_{k}\right)=\left[\begin{array}{c}
r_{k} \\
0
\end{array}\right]=\left[\begin{array}{c}
r_{k x} \\
r_{k y} \\
r_{k z} \\
0 \\
0 \\
0
\end{array}\right], \quad k=1, \cdots, n
$$

whose velocity states are zero, and where $n$ is the number of equilibria in the virtual net. See Figure 1. We assume that for all $r \in \mathcal{N}$, the corresponding values of control necessary to support the specified equilibria in steady-state satisfy the imposed thrust limits.

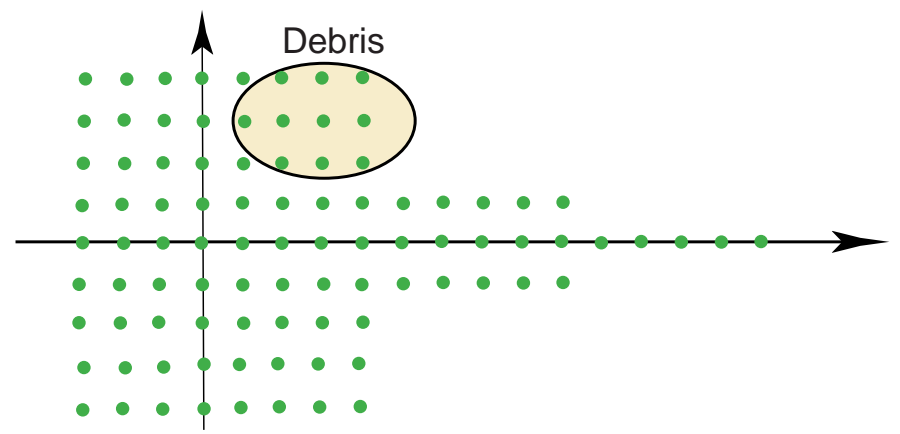

Fig. 1: The virtual net for debris avoidance. Dots correspond to positions at equilibria, $X_{e}(r)$, on a virtual net. The ellipsoid represents the debris position and uncertainty.

\section{B. LQ Controller with Gain Switching}

A conventional Linear-Quadratic (LQ) feedback is used to control the spacecraft to a commanded equilibrium in (4),

$$
U=K\left(X-X_{e}(r)\right)+\Gamma r=K X+H(K) r,
$$

where

$$
\begin{gathered}
\Gamma=\left[\begin{array}{ccc}
-3 n^{2} m_{c} & 0 & 0 \\
0 & 0 & 0 \\
0 & 0 & n^{2} m_{c}
\end{array}\right], \\
H(K)=\Gamma-K\left[\begin{array}{c}
I_{3} \\
0_{3}
\end{array}\right],
\end{gathered}
$$

and where $I_{3}$ denotes the $3 \times 3$ identity matrix and $0_{3}$ denotes the $3 \times 3$ zero matrix. This LQ controller provides an asymptotically stable closed-loop system but does not enforce the constraints.

To provide greater flexibility in handling constraints, a multimode controller architecture is employed [16]. Specifically, we assume that a finite set of LQ gains $K \in \mathcal{K}=\left\{K_{1}, \cdots, K_{m}\right\}$ is available to control the spacecraft. By using a large control weight in the LQ cost functional, motions with low fuel consumption yet large excursions can be generated; using a large control weight in the LQ cost, motions with short transition time can be generated [17]. We assume that a preference ordering has been defined and the gains are arranged in the order of descending preference, from $K_{1}$ being the highest preference gain to $K_{m}$ being the lowest preference gain.

\section{Positively Invariant Sets}

The ellipsoidal set

$$
\begin{aligned}
& \bar{C}(r, K)=\left\{X \in R^{6}:\right. \\
& \left.\frac{1}{2}\left(X-X_{e}(r)\right)^{T} P(K)\left(X-X_{e}(r)\right) \leq 1\right\} \subset R^{6},
\end{aligned}
$$


where $\bar{A}(K)^{\mathrm{T}} P \bar{A}(K)-P<0, \bar{A}(K)=(A+B K)$, $P=P(K)>0$, is positively invariant. Positive invariance implies that any trajectory of the closed-loop system that starts in $\bar{C}(r, K)$ is guaranteed to stay in $\bar{C}(r, K)$ as long as the same LQ gain $K$ is used and the same set-point command $r$ is maintained. To achieve the positive invariance, the matrix $P$ can be chosen as the solution of the discrete-time Riccati equation or of the above Lyapunov equation for the closed-loop asymptotically stable system. We note that, because the system is linear, the positive invariance of $\bar{C}(r, K)$ implies the positive invariance of the scaled set

$$
\begin{aligned}
& C(r, K, \rho)=\left\{X \in R^{6}:\right. \\
& \left.\frac{1}{2}\left(X-X_{e}(r)\right)^{T} P(K)\left(X-X_{e}(r)\right) \leq \rho^{2}\right\}, \quad \rho \geq 0 .
\end{aligned}
$$

Geometrically, the set $C(r, K, \rho)$ corresponds to an ellipsoid scaled by the value of $\rho$ and centered around $X_{e}(r), r \in \mathcal{N}$.

\section{Debris Representation}

We use a set, $O(z, Q)$, centered around the position $z \in R^{3}$, to over-bound the current position of the debris, i.e.,

$$
O(z, Q)=\left\{X \in R^{6}:(S X-z)^{T} Q(S X-z) \leq 1\right\}
$$

where $Q=Q^{\mathrm{T}}>0$ and

$$
S=\left[\begin{array}{llllll}
1 & 0 & 0 & 0 & 0 & 0 \\
0 & 1 & 0 & 0 & 0 & 0 \\
0 & 0 & 1 & 0 & 0 & 0
\end{array}\right]
$$

The set $O(z, Q)$ can account for the debris and spacecraft physical sizes and also for the uncertainties in the estimation of the debris/spacecraft position. Note that the set $O(z, Q)$ has an ellipsoidal shape in the position directions and it is unbounded in the velocity directions. Ellipsoidal sets rather than polyhedral sets are used to over-bound the debris since ellipsoidal bounds are typically produced by position estimation algorithms, such as the Extended Kalman Filter (EKF).

\section{E. Debris Avoidance Approach}

Consider now $r_{i} \in \mathcal{N}$, representing a possible position on the net that the spacecraft can move to as a part of the debris avoidance maneuver. Suppose first that the debris is stationary and that the current state of the spacecraft is $X\left(t_{0}\right)$ at the time instant $t_{0} \in Z^{+}$. If there exists a $\rho \geq 0$ and $K_{j} \in \mathcal{K}$ such that

$$
X\left(t_{0}\right) \in C\left(r_{i}, K_{j}, \rho\right) \text { and } O(z, Q) \cap C\left(r_{i}, K_{j}, \rho\right)=\emptyset \text {, }
$$

the spacecraft can move to the position $r_{i} \in \mathcal{N}$ by engaging the control law with $r(t)=r_{i}$ and $K(t)=$ $K_{j}, t \geq t_{0}$, and without hitting the debris confined to $O(z, Q)$. This idea underlies our approach to debris avoidance, in which we maintain the spacecraft trajectories within the tube formed by positively invariant sets that do not overlap with the debris.

To avoid a non-stationary debris, in [1], [2] we assume that its path can be covered by a union of a finite number of sets of ellipsoidal shape,

$$
\mathcal{D}=\bigcup_{l=1}^{l=n_{d}} O\left(z_{l}, Q_{l}\right),
$$

where the center of the $l$ th set is denoted by $z_{l} \in R^{3}$ and the $l$ th set shape is defined by $Q_{l}=Q_{l}^{\mathrm{T}}>0$. Then the debris path avoidance condition is

$$
\begin{gathered}
X\left(t_{0}\right) \in C\left(r_{i}, K_{j}, \rho\right) \text { and } \\
O\left(z_{l}, Q_{l}\right) \cap C\left(r_{i}, K_{j}, \rho\right)=\emptyset, \quad l=1, \ldots, n_{d} .
\end{gathered}
$$

The same approach, with larger $n_{d}$, can be used to handle multiple non-stationary debris. Note, however, that this approach is conservative as it does not account for the debris progressions along their paths versus time.

In this paper, we introduce the notion of time into the problem; whereas a transition between $r_{i}$ and $r_{j}$ might not be feasible at time $t_{1}$, based on the motion of a debris, it might become feasible at time $t_{2}$. To accommodate moving debris, we introduce a set $C_{k}(r, K, \rho), 0 \leq k \leq N$, defined by the following relation,

$$
\begin{gathered}
\bar{A}(K)^{k}\left(C_{k}(r, K, \rho)-\left\{X_{e}(r)\right\}\right) \\
=\left(C(r, K, \rho)-\left\{X_{e}(r)\right\}\right),
\end{gathered}
$$

Note that if $X(0) \in C_{k}(r, K, \rho)$, then $X(1) \in$ $C_{k-1}(r, K, \rho), X(2) \in C_{k-2}(r, K, \rho), \cdots, X(k) \in$ $C_{0}(r, K, \rho)=C(r, K, \rho)$.

\section{F. Connectivity Graph and Graph Search}

We now introduce a notion of connectivity between two vertices of the virtual net, $r_{i} \in \mathcal{N}$ and $r_{j} \in \mathcal{N}$ at a specified time $t_{0}$. This notion is based on the fact that the time to transition from any state in $C_{N}(r, K, \rho)$ to $C(r, K, \rho)$ is less or equal than $N$ steps. Suppose that the debris path $D\left(t_{0}: t_{0}+N \cdot H\right)$ has been predicted over the $N \cdot H$ discrete steps from the time instant $t_{0}$, where

$$
D\left(t_{k}: t_{r}\right)=\bigcup_{t=t_{k}}^{t=t_{r}} O(z(t), Q(t)) .
$$


The node $r_{i} \in \mathcal{N}$ is connected to $r_{i} \in \mathcal{N}$ at the time instant $t_{k}=t_{0}+k N$ if there exists $K \in \mathcal{K}$ such that

$$
D\left(t_{k}: t_{k}+N\right) \cap C\left(r_{i}, K, \rho\right)=\{\emptyset\} .
$$

The node $r_{i} \in \mathcal{N}$ is connected to node $r_{j} \in \mathcal{N}$ at time $t_{k}$ if there exists $K \in \mathcal{K}$ such that

$$
\begin{aligned}
& D\left(t_{k}: t_{k}+N\right) \cap C_{N}\left(r_{j}, K, \rho\right)=\{\emptyset\} \\
& \text { and } \\
& C\left(r_{i}, K, \rho\right) \subset C_{N}\left(r_{j}, K, \rho\right) .
\end{aligned}
$$

The connectivity implies that a spacecraft located close to an equilibrium corresponding to $r_{i}, X_{e}\left(r_{i}\right)$, can transition to an equilibrium $X_{e}\left(r_{j}\right)$ between the time instants $t_{k}$ and $t_{k}+N$ while avoiding collision with the debris. We note that if $r_{i}$ is connected to $r_{j}$ this does not imply that, in turn, $r_{j}$ is connected to $r_{i}$. We also note that connectivity depends on the existence of an appropriate control gain from the set of gains $\mathcal{K}$ but does not need to hold for all gains. Furthermore, since connectivity depends on the predicted motion of the debris, connectivity is time-varying.

The on-line motion planning with debris avoidance is performed according to the following procedure:

Step 1: Determine the debris location, shape and predict the debris path $D\left(t_{0}: t_{0}+N \cdot H\right)$

Step 2: Construct graph connectivity matrices corresponding to $t_{k}, k=0,1, \cdots, H$. In the graph connectivity matrix, if two vertices, $r_{i}$ and $r_{j}$, are not connected at $t_{k}$, the corresponding matrix element is zero; if they are connected the corresponding matrix element is 1 . In parallel, build the control gain selectivity matrix, which identifies the index of the highest preference gain $K$ for which $r_{i}$ and $r_{j}$ are connected. This gain will be applied if the edge connecting $r_{i}$ and $r_{j}$ is traversed.

Step 3: Perform graph search to determine a sequence $r\left[t_{k}\right] \in \mathcal{N}$ and control gains $K[k] \in$ $\mathcal{K}, k=1, \cdots, l_{p}$, such that $r\left[t_{1}\right]$ satisfies the initial constraints, $r\left[l_{p}\right]$ satisfies the final constraints, and the path length $l_{p}$ (or another cost function such as the expected fuel consumption) is minimized.

Per the above algorithm, a graph search is utilized to determine the minimum number of equilibrium hops around a debris starting at $t_{0}$.

Remark 1: The condition (15) is conservative. It can be replaced by a less conservative condition,

$$
\begin{gathered}
D\left(t_{k}: t_{k}+m\right) \cap C_{N-m}\left(r_{j}, K, \rho\right)=\{\emptyset\}, \\
m=0,1, \cdots, N,
\end{gathered}
$$

at a price of more demanding computations.

Remark 2: The condition (15) is checked computationally using the fast growth distance algorithm described in [1], [2]. The intersection is empty if $C_{N}$ can be grown before it touches $D\left(t_{k}: t_{k}+N\right)$. This fast growth distance algorithm is essential to be able to rapidly construct the connectivity matrices.

Remark 3: In our simulations, the path search is performed using the standard Dijkstra's algorithm. It is applied to a lifted graph with vertices being the pairs $\left(r_{i}, t_{k}\right)$.

\section{Simulation Results}

Simulations are now provided that illustrate our debris avoidance approach, and contrast it with our prior results in [1], [2]. We consider a nominal circular orbit of $850 \mathrm{~km}$ and discretize the $\mathrm{HCW}$ equations with a sampling period, $\Delta T=30 \mathrm{sec}$. We construct an approximately $2 \mathrm{~km}$ cubed virtual net. We let $R=$ $2 \times 10^{7} I_{3}$ and $Q=\operatorname{diag}\left(100,100,100,10^{7}, 10^{7}, 10^{7}\right)$, $\mathcal{K}=K_{1}$.

We first consider the case of a non-stationary debris where we treat its motion as the union of static debris along the path as described in [2]. A union of ellipsoidal sets over-bounds the debris' motion, $\bigcup_{l=1}^{l=n_{d}} O\left(z_{i}, Q_{i}\right)$, where $z_{i}$ are generated by sampling the relative motion of the debris with the initial condition $\left[\begin{array}{lllll}0 & 0.5 & 0 & 0 & 0.0006\end{array}\right]^{\mathrm{T}}$, and with $Q_{i}=200 I_{3}, i=$ $1 \ldots n_{d}$. The spacecraft's initial condition is $X(0)=$ $X_{e}\left(r_{0}\right)$, where $r_{0}=\left[\begin{array}{lll}0 & 1 & 0\end{array}\right]^{\mathrm{T}}$. The target equilibrium node is $X_{e}(0)$. We impose a maximum thrust constraint of $10 \mathrm{~N}$ in each axis. Dijkstra's algorithm is used to find the shortest length path from the initial node to the final node. The simulation results in Figure 2 demonstrate the the spacecraft is able to avoid the closed debris path by hopping over it.

We now repeat this simulations using the approach described in this paper which takes into account the debris' motion as a function of time. Figure 2 shows that the graph search algorithm is able to find a path which passes through the debris' path but avoids collision due to the debris' location elsewhere at the specific time instant at which the spacecraft path crosses the debris' path.

Finally, Figure 4 demonstrates another maneuver of avoiding a debris, following a different path. Note that the thrust does not converge to zero since the target spacecraft position is not an in-track equilibrium.

\section{CONCLUSION}

This paper described an approach to spacecraft maneuver planning that uses contractive constraint admis- 


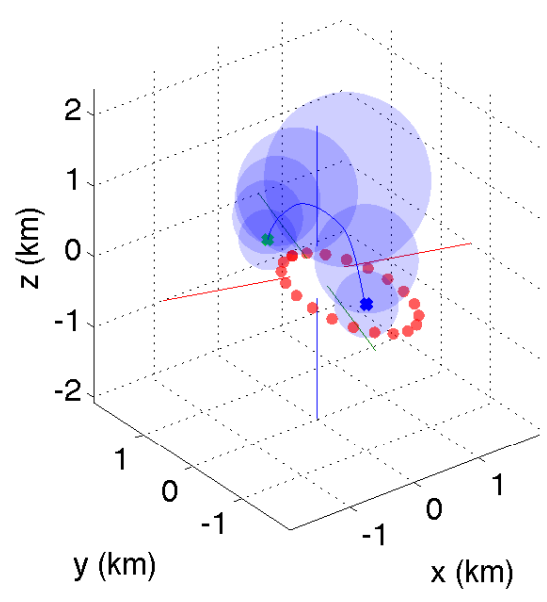

(a) Debris Avoidance Path.

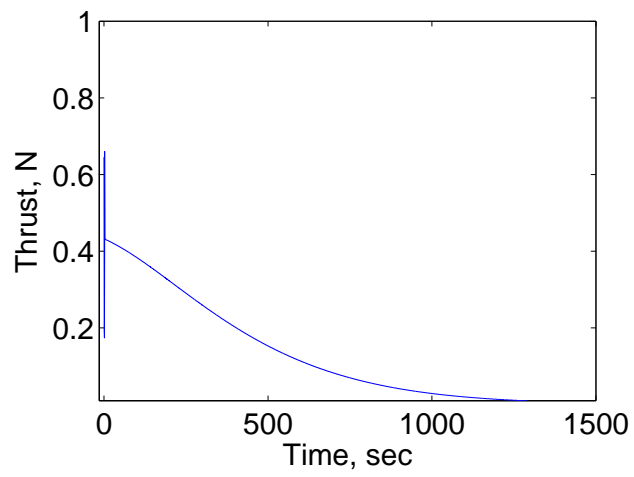

(b) Norm of Thrust Profile.

Fig. 2: (a) Debris avoidance path for a non-stationary debris using the union method described in [2]. The green $\mathrm{x}$ marks the initial node. The blue $\mathrm{x}$ marks the final node. The red ellipsoids represent the debris path. The blue line is the path the spacecraft takes in order to avoid the debris. The blue ellipsoids represent the maximally grown (see [2]) invariant sets, $C$, along the path. (b) The time history of thrust magnitude.

sible sets in order to avoid collisions with a moving debris. Future work will explore cost functions other than the path length and include set-bounded uncertainty into the motion planning.

\section{REFERENCES}

[1] Baldwin, M., Weiss, A., Kolmanovsky, I., and Erwin, R.S.,"Spacecraft Debris Avoidance using Constraint Admissible Positively Invariant Sets," Proceedings of AAS Space Flight Mechanics Meeting, Paper AAS 12-250, Charleston, South Carolina, 2012.

[2] Weiss, A., Baldwin, M., Erwin, R.S., and Kolmanovsky, I.,"Spacecraft Constrained Maneuver Planning using Positively Invariant Constraint Admissible Sets," Proceedings of Bar-Itzhack Memorial Symposium, Haifa, Israel, October 2012.

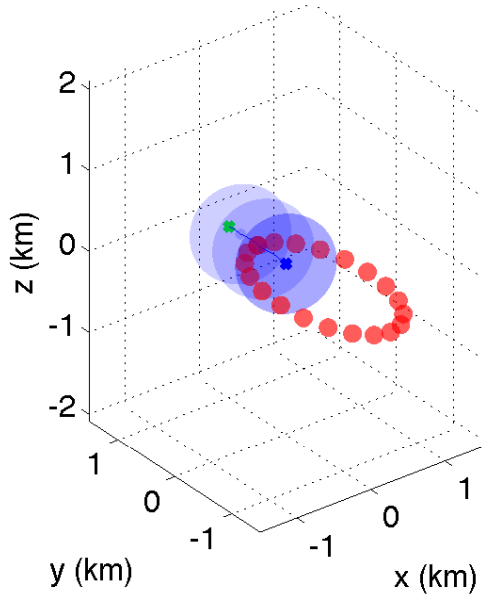

(a) Debris Avoidance Path.

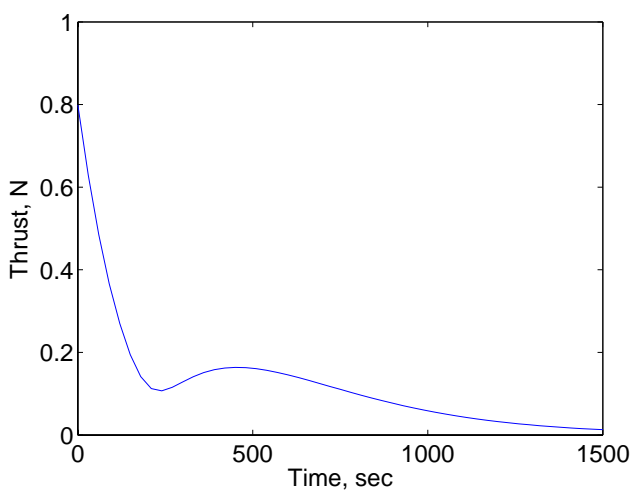

(b) Norm of Thrust Profile.

Fig. 3: (a) Debris avoidance path for a non-stationary debris using the contractive set approach described in this paper. The green $x$ marks the initial node. The blue $\mathrm{x}$ marks the final node. The red ellipsoids represent the debris path. The blue line is the path the spacecraft takes in order to avoid the debris. The blue ellipsoids represent the invariant sets, $C_{N}$, along the path. (b) The time history of thrust magnitude.

[3] LaValle, S.M., Planning Algorithms, Cambridge University Press, 1996.

[4] Maia, M.H. and Galvao, R.K.H., "On the use of mixedinteger linear predictive control with avoidance constraints," International Journal of Robust and Nonlinear Control, Vol. 19, pp. 822-828.

[5] Ranieri, C"Path-constrained trajectory optimization for proximity operations," AIAA/AAS Astrodynamics Specialist Conference, Honolulu, HI, August 2008.

[6] Epenoy, R., "Fuel optimization for continuous-thrust orbital rendezvous with collision avoidance constraint," Journal of Guidance, Control, and Dynamics, Vol. 34, No. 2, MarchApril 2011.

[7] Sanchez-Ortiz, N., Bello-Mora, M., and Klinkrad, H., "Collision avoidance manoeuvres during spacecraft mission lifetime: Risk reduction and required $\Delta \mathrm{V}$," Advances in Space Research, Vol. 38, 2006. 


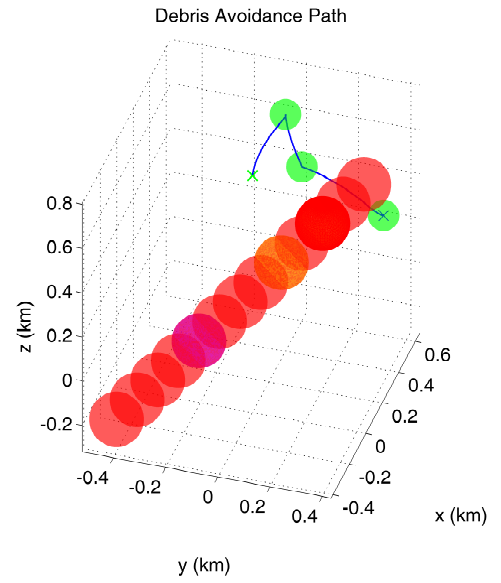

(a) Debris Avoidance Path.

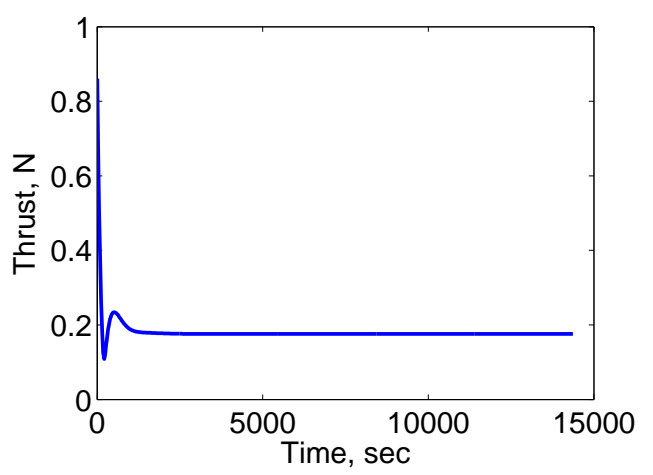

(b) Norm of Thrust Profile.

Fig. 4: (a) Debris avoidance path for a non-stationary debris using the contractive set approach described in this paper. The green $x$ marks the initial node. The blue $x$ marks the final node. The red ellipsoids represent the debris path. The blue line is the path the spacecraft takes in order to avoid the debris. The green ellipsoids represent the sets, $C=C_{0}$, along the spacecraft path. (b) The time history of thrust magnitude.

[8] Martinson, N., Munoz, J., and Wiens, G., "A new method of guidance control for autonomous rendezvous in a cluttered space environment," Proceedings of AIAA Guidance, Navigation, and Control Conference, Hilton Head, SC, August 2007.

[9] Martinson, N.,"Obstacle avoidance guidance and control algorithms for spacecraft maneuvers," Proceedings of AIAA Guidance, Navigation, and Control Conference, Chicago, IL, August 2009.

[10] Mueller, J., Griesemer, P., and Thomas, S., "Avoidance maneuver planning incorporating station-keeping constraints and automatic relaxation," AIAA Infotech@Aerospace 2010, Atlanta, GA, April 2010.

[11] Ulybyshev, Y., "Trajectory optimization for spacecraft proximity operations with constraints," AIAA Guidance, Navigation, and Control Conference, Portland, OR, August 2011.

[12] Richards, A., Schouwenaars, T., How, J., and Feron, E., "Spacecraft trajectory planning with avoidance constraints using mixed-integer linear programming," AIAA Journal of Guidance, Control, and Dynamics, Vol. 25, No. 4, August 2002.
[13] Mueller, J.,"Onboard planning of collision avoidance maneuvers using robust optimization," AIAA Infotech@Aerospace 2009, Seattle, WA, April 2009.

[14] Kolmanovsky I.V., Gilbert E.G., "Theory and computation of disturbance invariant sets for discrete-time linear systems," Mathematical Problems in Engineering, Vol. 4(4), pp. 317367, 1998.

[15] Wie, B., Spacecraft Dynamics and Control, 2nd Ed. Reston, VA: AIAA,2010.

[16] Kolmanovsky, I.V., and Gilbert, E., "Multimode regulators for systems with state and control constraints and disturbance inputs," Proceedings of Workshop Control Using Logic-Based Switching, pp. 104-117, published in an edited volume by Springer-Verlag, Editor: Morse A.S, Block Island, Rhode Island, Fall, 1997.

[17] Park, H., Di Cairano, S., and Kolmanovsky, I.V., "Linear Quadratic Model Predictive Control approach to spacecraft rendezvous and docking," Proceedings of 21st AAS/AIAA Space Flight Mechanics Meeting, New Orleans, Louisiana, USA, Paper AAS-142, 2011.

[18] Ong C.J., Gilbert E.G., "Growth distances: New measures for object separation and penetration," IEEE Transactions on Robotics and Automation, Vol. 12(6), pp. 888-903, 1996. 